\title{
Transcriptomic profiling fuels the derivation of stable pig epiblast stem cells
}

\author{
Cuiqing Zhong ${ }^{1,3}$, Ronghui $\mathrm{Li}^{2,3}$ and Juan Carlos Izpisua Belmonte $\mathbb{D}^{1 凶}$
}

(c) CEMCS, CAS 2022

Cell Research (2022) 32:329-330; https://doi.org/10.1038/s41422-021-00609-3

\begin{abstract}
It is controversial whether pig pluripotent stem cells can be derived and stably maintained in a culture dish. In a recent paper published in Cell Research, Zhi et al. perform a comprehensive transcriptome landscape analysis of pig embryos at all developmental stages from embryonic (E) day 0 to E14 and identify a culture cocktail to stabilize pig pregastrulation epiblast stem cells (pgEpiSCs) in vitro for long-term passaging; the pgEpiSCs can undergo multiple rounds of gene editing and generate cloned animals.
\end{abstract}

Pluripotent stem cells (PSCs), which can develop into three differentiated embryonic germ layers, have been successfully derived in mammals from pre-implantation embryos to gastrulation embryos. Mouse embryonic stem cells (ESCs) derived from the blastocyst represent a naïve state of pluripotency at the preimplantation stage. ${ }^{1}$ Primed PSCs are the in vitro counterpart of a later developmental stage of epiblast cells in post-implantation embryos. ${ }^{2}$ Mouse epiblast stem cells, conventional human ESCs, or human induced PSCs were deemed as primed PSCs. ${ }^{3}$ Both naïve and primed PSCs have been used extensively to study early embryonic development and to develop cell replacement therapies for the treatment of a variety of degenerative diseases. ${ }^{4}$

Pigs as a research animal model have significant advantages over rodents due to their greater similarity to humans in terms of organ size, early post-implantation development, and physiology, among other qualities. Although a variety of mouse and human PSCs have been generated from pre-implantation and postimplantation embryos, there are difficulties in stabilizing the pluripotent state in many livestock species. ${ }^{5}$ Despite years of research, successful long-term cultivation of pig PSCs remains an unresolved challenge that hinders their potential application for gene editing, disease modeling, and regenerative medicine.

To comprehensively understand the molecular signaling of pig embryogenesis, Zhi et al. $^{6}$ systematically characterized the transcriptomic landscape in pig embryos from E0 to E14 at the single-cell level. This rich dataset provides a valuable resource for the scientific community to further investigate embryonic development and to determine species-specific developmental factors. Based on these single-cell RNA sequencing (scRNA-seq) data, Zhi et al. showed that the lineage segregation and signature expressions during pig embryo development are consistent with previous studies. To elucidate the reasons for the difficulty and failure in capturing the naïve and primed pluripotent states in pigs, the authors performed a further inspection of the signaling pathways required for pluripotency and differentiation in E0-E14 embryos and suggested that the maintenance of the pig epiblast in vitro requires both the presence of the activin and FGF pathways and the inhibition of the Wnt and JAK/STAT3 pathways. Interestingly, the scRNA-seq analysis also revealed a dramatic loss of naïve pluripotency in pigs as early as the morula (E4) to the blastocyst (E7), whereas epiblasts maintain a steady formative state from E7 to E10, suggesting that stable PSC lines are more likely to be established in pigs at these stages.

To this end, Zhi et al. applied the defined cytokines or inhibitors to target these signaling pathways and determined the optimized condition " $3 \mathrm{i} / \mathrm{LAF}$ " for in vitro pig pregastrulation epiblast stem cells (pgEpiSCs) to fully capture pluripotency of the in vivo epiblast of E10 embryos, which sustains the capability of pgEpiSCs to selfrenew and differentiate into three germ layers even after longterm passaging. Global transcriptome analysis confirmed that pgEpiSCs resemble their in vivo pregastrulation epiblast counterparts. To further characterize the chromatin architecture of pgEpiSCs, the authors performed $\mathrm{HiC}$-seq and ATAC-seq and found that pgEpiSCs possess a looser chromatin structure and higher accessibility to pluripotency modules compared to pig embryonic fibroblasts. To examine the function of pgEpiSCs in the developing embryo, the authors injected them into E5 early blastulae. They observed a very limited contribution of pgEpiSCs in chimeras, which might be due to the incompatibility of pgEpiSCs in the blastocyst environment.

To explore the potential application of pgEpiSCs in genome selection and disease modeling, Zhi et al. conducted several rounds of gene editing in pgEpiSCs and used them as donors for cloning (Fig. 1). The cloned embryos were transferred into the uteruses of surrogates to assess their developmental potential. Notably, the cloning efficiency of gene-edited pgEpiSCs is equivalent to those of wild-type pgEpiSCs and embryonic fibroblasts, highlighting its potential as a new avenue for genomic selection of husbandry and regenerative medicine.

The study by Zhi et al. sparks many intriguing questions. For instance, can the culture condition and protocol developed in this study be applied to generate stable EpiSCs in other domesticated animal species? Additionally, it will be interesting to elucidate and verify the specific signatures of E0-E7 pig embryos that are distinct from other species by analyzing these scRNA-Seq data to uncover their own regulatory mechanisms for early lineage specification and commitment, and to shed light on the

\footnotetext{
${ }^{1}$ Altos Labs, San Diego, CA, USA. ${ }^{2}$ Salk Institute for Biological Studies, La Jolla, CA, USA. ${ }^{3}$ These authors contributed equally: Cuiqing Zhong, Ronghui Li.

email: jcbelmonte@altoslabs.com
} 


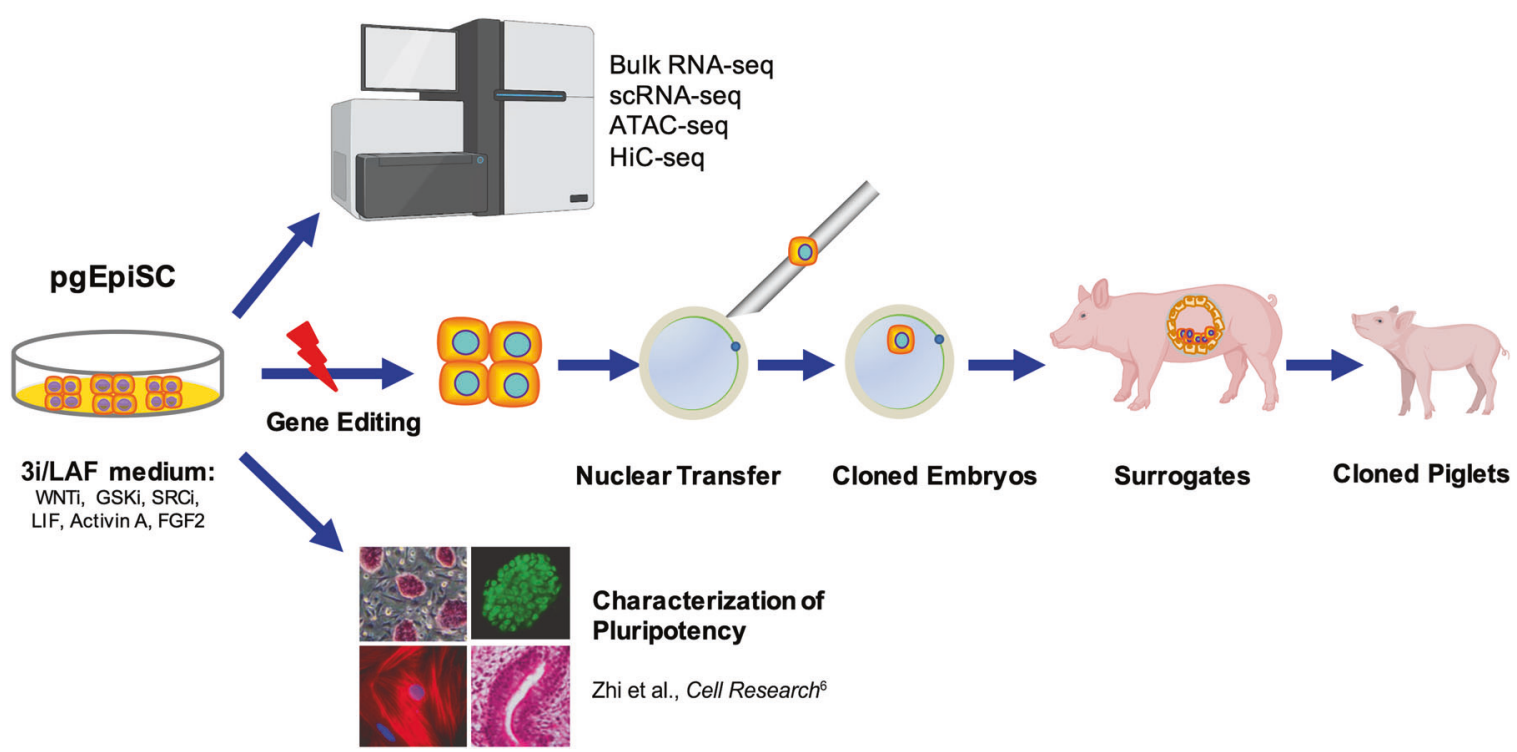

Fig. 1 Summary of pgEpiSC derivation and its application to the generation of gene-edited pigs. pgEpiSCs were cultured in $3 \mathrm{i} / \mathrm{LAF}$ medium and further characterized for the expression of pluripotency markers and the potential to differentiate into three germ layers (lower). Multi-omics sequencing was performed to identify transcriptome and chromatin architecture features in pgEpiSCs (upper). pgEpiSCs can be used as a donor for nuclear transfer to produce cloned piglets, even after multiple rounds of gene editing (middle).

development of culture conditions to capture stable naïve pluripotency in the pig.

Overall, the data presented by Zhi and colleagues broaden our understanding of the pre- and post-implantation development of pig embryos, enable very long-term cultivation of pig PSCs in the dish, facilitate the generation of multiple loci-edited cloned piglets, and greatly accelerate the generation of human disease models in pigs as well as the creation of genetically superior pigs, etc. The pgEpiSCs could also be used to understand the interaction between pig cells and primate cells in co-culture experiments, which will facilitate research of generating human organs in pigs. 7,8

\section{REFERENCES}

1. Ying, Q.-L. et al. Nature 453, 519-523 (2008).

2. Nichols, J. \& Smith, A. Cell Stem Cell 4, 487-492 (2009).
3. Wu, J. \& Izpisua Belmonte, J. C. Cell Stem Cell 17, 509-525 (2015).

4. Yamanaka, S. Cell Stem Cell 27, 523-531 (2020).

5. Ezashi, T., Yuan, Y. \& Roberts, R. M. Annu. Rev. Anim. Biosci. 4, 223-253 (2016).

6. Zhi, M. et al. Cell Res. https://doi.org/10.1038/s41422-021-00592-9 (2021).

7. Wu, J. et al. Cell 168, 473-486.e15 (2017).

8. Tan, T. et al. Cell 184, 2020-2032 (2021).

\section{COMPETING INTERESTS}

The authors declare no competing interests.

\section{ADDITIONAL INFORMATION}

Correspondence and requests for materials should be addressed to Juan Carlos Izpisua Belmonte.

Reprints and permission information is available at http://www.nature.com/ reprints 